\title{
Incidental Findings of Caesarean Section Scar Defect - Case Reports and a Review of its Investigations and Management
}

Felix Wong Wu Shun*, Eric Lee Tat Choi and Belinda Leung Fung $\mathrm{Ha}$

Hong Kong Sanatorium and Hospital, Hong Kong

\begin{abstract}
The clinical awareness of Caesarean scar defect (CSD) as a cause of abnormal uterine bleeding and adverse obstetrical outcome is still lacking among gynaecologists and their patients. Patients with CSD are mostly asymptomatic, though the lesion may be associated with symptoms like abnormal uterine bleeding, pelvic pain, infertility, and adverse pregnancy outcome. This paper is to report two patients with incidental findings of CSD on ultrasound scanning and MRI and to discuss on their presentations, investigations, and dilemma on treatment options of this condition.
\end{abstract}

Keywords: MRI image; Ultrasound image; Caesarean scar defect (CSD); Abnormal uterine bleeding

\section{Introduction}

Incidence of Caesarean scar defect is expected be on the increase as there is a rising trend of Caesarean sections performed nowadays. Despite its prevalence, the clinical awareness of Caesarean scar defect (CSD) as a cause of abnormal uterine bleeding, pelvic pain, infertility and uterine dehiscence and rupture is largely lacking. Furthermore, the clinical implications of this condition on cervical or CS scar ectopic pregnancy and uterine rupture are often overlooked by most gynaecologists. Ultrasound scan is commonly performed in gynaecological practice and has been shown to be useful in diagnosing CSD as reported in the literature [1-3]. However, the sensitivity of ultrasound scan in diagnosing a CS scar defect depends on the expertise of the operator and whether a deliberate search for this condition is made at the time of the scanning. MRI, on the other hand, is far less commonly employed to investigate gynaecological problems because of its associated cost and is primarily ordered only for a special indication. Images of MRI on CSD are, therefore, not as often reported in the literature and they are found mainly in studies done by groups who are interested in CSD [4]. As MRI of the pelvis is sometimes performed for various other reasons, incidental finding of CSD as in one of our cases will raise an issue on the management of this condition. This paper is to describe the incidental ultrasound and MRI findings of this anatomic defect in the lower anterior uterine wall in two women with previous Caesarean section, and to discuss on its imaging investigations and management.

\section{Case Report}

Case 1: The patient is a 41 year old gravida 1 para 1 woman with history of dysmenorrhea, prolonged periods lasting for 8-10 days, and postmenstrual spotting. She had undergone a lower segment Caesarean section together with the removal of a left ovarian endometriotic cyst 6 years previously. She presented with progressive lower abdominal pain and had received ultrasound scanning to investigate the reason behind the pain. The ultrasound examination revealed a right ovarian tumour measuring at $8.1 \mathrm{~cm} \times 5.2 \mathrm{~cm}$ with solid and cystic components. Magnetic Resonant Imaging (MRI) of the pelvis was performed to further define the nature of her ovarian tumour prior to surgery. The MRI investigation was carried out while she was on the last day of her menstrual period and it confirmed the presence of a right ovarian cyst with signals suggestive of either haemorrhage or high protein content within the cyst, but absence of any solid component. Incidentally, the MRI also showed a cystic collection over the lower portion of the anterior uterine wall close to the level of the cervix (Figure 1). The remaining thickness of the uterine wall corresponded to site of the lesion was much reduced. The impression given was that of a Caesarean section scar defect.

She was then scheduled for a diagnostic hysteroscopy and laparoscopic right ovarian cystectomy in January 2015. At laparoscopy, a right ovarian endometriotic cyst was confirmed and ovarian cystectomy was performed. Hysteroscopic examination revealed the presence of retained blood clots in a niche over the lower anterior uterine wall (Figure 2). A CSD was diagnosed, but no surgical correction of the defect was made because that was not requested by the patient though the implications of the MRI findings had been discussed prior to the operation. The patient recovered well and was discharged home on day 2 after the surgery. At her follow up visit, she was informed of the Caesarean scar defect which could possibly account for her prolonged periods. As she was not considering future pregnancy, she was prescribed oral contraceptive pills to regulate and minimize her periods as well as for contraceptive purpose. Her subsequent periods following treatment were shortened to 3-5 days and she had since remained symptom free.

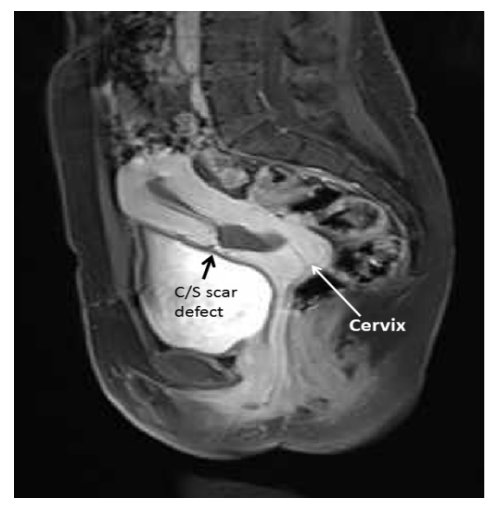

Figure 1: MRI image of CS scar defect.

*Corresponding author: Felix Wong Wu Shun, Hong Kong Sanatorium and Hospital Hong Kong, Tel: 3905 3905; E-mail: fwong3@hotmail.com.hk

Received November 01, 2015; Accepted December 05, 2015; Published December 12 2015

Citation: Wu Shun FW, Choi ELT, Fung Ha BL (2015) Incidental Findings of Caesarean Section Scar Defect - Case Reports and a Review of its Investigations and Management. J Clin Case Rep 5: 674. doi:10.4172/2165-7920.1000674

Copyright: (c) 2015 Wu Shun FW, et al. This is an open-access article distributed under the terms of the Creative Commons Attribution License, which permits unrestricted use, distribution, and reproduction in any medium, provided the original author and source are credited. 
Citation: Wu Shun FW, Choi ELT, Fung Ha BL (2015) Incidental Findings of Caesarean Section Scar Defect - Case Reports and a Review of its Investigations and Management. J Clin Case Rep 5: 674. doi:10.4172/2165-7920.1000674

Page 2 of 3

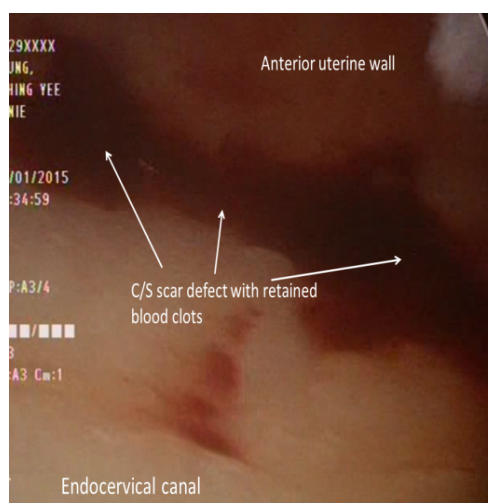

Figure 2: Hysteroscopic picture of CS scar defect with retained blood clots.

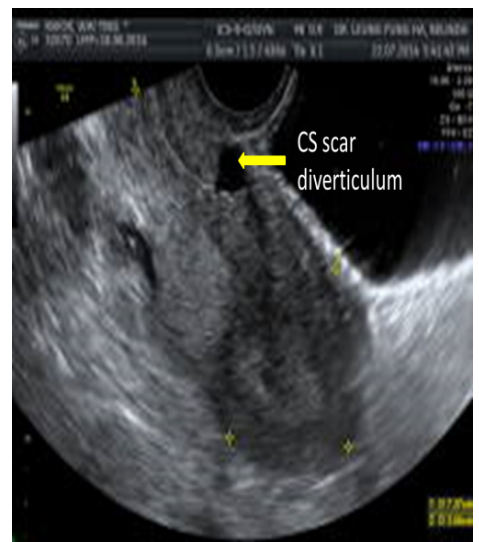

Figure 3: Ultrasound image of CS scar diverticulum at anterior lower uterine wall.

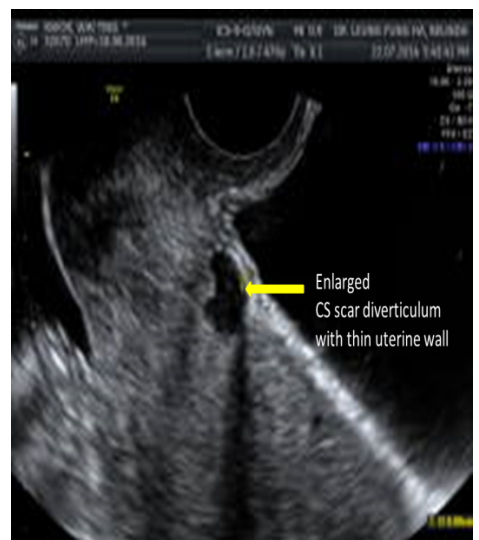

Figure 4: Enlarged ultrasound image of CS scar diverticulum with thin uterine wall.

Case 2: The patient is a 30 year old gravida 2 para 1 woman with history of Caesarean section performed in 2013. She had a spontaneous early complete miscarriage in June 2014. After her miscarriage, an ultrasound scan was performed and it revealed an obvious CSD with only a very thin layer of remaining myometrial tissue identified (Figures 3 and 4). She was informed of the potential risks associated with the CSD including that of Caesarean scar ectopic pregnancy, placenta accreta and uterine rupture in her future pregnancy. As she intended to have another baby, she came for medical advice and finally decided to go for surgical repair of the defect.

Clinically, she also noticed intermenstrual bleeding for which she had accepted as normal since her last CS delivery. She was then admitted to hospital for a diagnostic hysteroscopy and laparoscopic repair to her CS scar defect. Diagnostic hysteroscopy was performed and confirmed a deep scar defect filled with retained blood clots. Laparoscopic surgical repair of the defect was carried out. The surgical techniques employed involved temporary bilateral uterine artery occlusion, bladder dissection for enhancing exposure, identification and delineation of the entire CS scar defect, total excision of the CS scar, and a double layered repair of the resulting uterine wound under direct laparoscopic visualization (Figure 5).

The patient recovered well after her operation and was discharged home on the second postoperative day without complication. An ultrasound scan performed on her follow up visit showed normal thickness of the uterine wall over the site of the previous CS scar, and only a tiny shallow defect was left detectable (Figure 6). She was told that the risks of adverse pregnancy outcome could likely to be reduced, but careful ultrasound monitoring of the scar thickness throughout pregnancy was necessary and repeat LSCS would be advisable.

\section{Discussion}

Caesarean Scar Defect (CSD) is also known as Caesarean section scar diverticulum, Caesarean section scar niche, Caesarean section scar dehiscence, or Caesarean hysterotomy scar defect. As Caesarean sections are now being increasingly accepted by patients as a preferred mode of delivery and the escalating medico-legal liability associated with vaginal delivery in developed countries, there is a rising trend on the use of Lower Segment Caesarean Section and thus possibly leading to an expected concomitant increase in the incidence of CSD. The incidence of CSD has been reported to be as high as $60 \%$ of cases [1]. A systemic literature review on women who had undergone previous Caesarean section as evaluated by hysterography, sonohysterography (SHG), or transvaginal ultrasound (TVU), demonstrated the presence of uterine scar defects in $50 \%$ of cases [2]. Therefore, physicians should

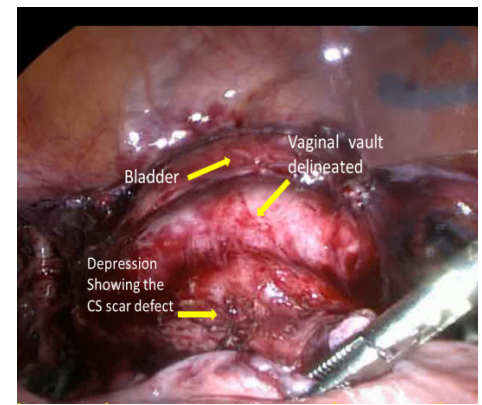

Figure 5: Laparoscopic picture of CS scar defect showing depressed scar wall.

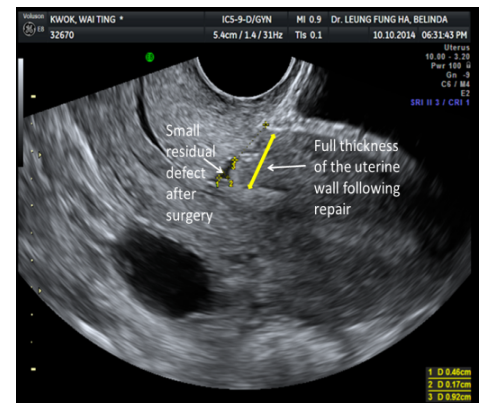

Figure 6: Ultrasound image of CS scar showing thickened uterine wall with a small defect after laparoscopic surgical repair of CS scar defect. 
be on the alert and have a high index of suspicions of this condition when managing women with previous Caesarean section.

Investigations of this condition were commonly done by using ultrasonogram [3]. Armstrong and Hansen 2003 claimed that transvaginal ultrasound was highly accurate in detecting Caesarean scar defect and they defined it as the presence of fluid within the scar site [4]. Many studies had advocated the use of ultrasound investigation in the diagnosis and assessment of CSD [5-7]. Tower and Frishman proposed that a CSD can be diagnosed through transvaginal saline sonohysterography by revealing a triangular hypoechoic defect in the myometrium at the site of the previous hysterotomy scar [8]. Although there have been many reports on this condition, many inexperienced gynaecologists could still miss the defect during routine ultrasound scanning because of a lack of high index of suspicion.

Hysterosalpingography is commonly used to investigate patients with infertility and it can also demonstrate the presence of a CS scar defect. Surapaneni and Silberzweig defined the CS scar defect as a diverticulum at the lower uterine cavity, uterine isthmus, or upper endocervical canal on hystero-salpingography. In their study of 148 patients with previous CS and infertility, $60 \%$ of their patients had defects showing anterior uterine diverticula. Fifty-eight (65\%) of the diverticula were focal outpouchings, and 31 (35\%) were thin linear defects [9].

MRI as the investigative imaging tool in the diagnosis of CS scar defect is not widely reported because it is not usually the priority investigation done for women with abnormal uterine bleeding or other uterine abnormality. It would not be a surprise for radiologists not reporting on the condition as a defect in the uterus even on MRI study because it is simply not being asked for. MRI is costly but it can accurately define the CS scar lesions. If incidental finding of this condition is noted in an MRI investigation for a unrelated condition, the management may vary depending on the symptoms and the demand for future pregnancy.

Incidental finding of a CS scar defect can pose a management problem for women who have undergone MRI or ultrasound investigations for other reasons. As in our first patient who complained of prolonged menses and pain, she preferred not to receive surgical repair to the defect despite the CSD was being diagnosed prior to her operation. She made such a decision because the prolonged menstrual bleeding was relatively acceptable and she did not anticipate future pregnancy. She was treated with good response using hormonal therapy. For patients with CSD presenting with postmenstrual spotting and pain, hysteroscopic resection of the CSD niche at the time of hysteroscopic evaluation may result in alleviation of symptoms. Vervoort et al., reported a multicentre randomised trial (Dutch Trial NTR3269 - registered in February 2012) that might provide evidence on the cost effectiveness of hysteroscopic resection versus expectant management for symptomatic patients [10]. A conclusion drawn on the cost effectiveness analysis is expected to be available to guide the management of symptomatic patients who have no desire for pregnancy by the end of the trial.

The approach of the surgical treatment can vary and it depends on the symptomatology, severity of the defect, risk of uterine rupture, and the desire for future pregnancy. Both hysteroscopic or laparoscopic surgery have been reported to be useful in the management of this condition [11]. The excision of the inflamed scar tissue may be performed via laparoscopic, combined laparoscopic-vaginal, vaginal, or hysteroscopic approach. Recently, Api et al., are of the opinion that hysteroscopic treatment like resection of the CSD niche can only solve the problem of abnormal uterine bleeding but not any pregnancy related sequelae. They advocated the use of laparoscopic repair which can provide both the correction of abnormal bleeding as well as restoration of thickness of the involved uterine wall and thus reducing the risk of uterine dehiscence for those who intend to go for future pregnancy [12]. This is in accordance to the rationale behind the recommendation that we had given to our second patient.

It is still controversial at present as to when, how and what treatment should be offered to our patients with CSD. There is a lack of large series study or prospective randomised trials to determine if treatment to the condition is warranted, the best treatment modality, carries lowered rate of CS scar pregnancies, improved fertility, and reduced risk of uterine rupture in pregnancy after surgical correction. On the other hand, one is yet to decide whether there would be an acceptable risk of rupture without going for surgical repair. Hopefully, with increasing awareness and identification of this condition, larger studies can be performed to clarify the issue.

\section{Conclusion}

Both MRI and ultrasound scan are useful investigations to detect and define the extent of Caesarean scar defect (CSD) and they can contribute to the management of the condition by providing an accurate diagnosis which can help to guide the treatment and predict the clinical implications and future pregnancy outcome.

\section{References}

1. Osser OV, Jokubkiene L, Valentin L (2009) High prevalence of defects in Cesarean section scars at transvaginal ultrasound examination. Ultrasound Obstet Gynecol 34: 90-97.

2. Armstrong V, Hansen WF, Van Voorhis BJ, Syrop CH (2003) Detection of cesarean scars by transvaginal ultrasound. Obstet Gynecol 101: 61-65.

3. Mohammed ABF (2010) Ultrasonographic evaluation of lower uterine segment thickness in pregnant women with previous cesarean section. Middle East Fertility Society Journal 1: 88-193.

4. Donnez O, Jadoul P, Squifflet J, Donnez J (2008) Laparoscopic repair of wide and deep uterine scar dehiscence after cesarean section. Fertil Steril 89: 974980.

5. Roberge S, Boutin A, Chaillet N, Moore L, Jastrow N, et al. (2012) Systematic review of cesarean scar assessment in the nonpregnant state: imaging techniques and uterine scar defect. Am J Perinatol 29: 465-471.

6. Thurmond AS, Harvey WJ, Smith SA (1999) Cesarean section scar as a cause of abnormal vaginal bleeding: diagnosis by sonohysterography. J Ultrasound Med 18: 13-16.

7. Naji O, Abdallah Y, Bij De Vaate AJ, Smith A, Pexsters A, et al. (2012) Standardized approach for imaging and measuring Cesarean section scars using ultrasonography. Ultrasound Obstet Gynecol 39: 252-259.

8. Tower AM, Frishman GN. (2013) Cesarean scar defects: an underrecognized cause of abnormal uterine bleeding and other gynecologic complications. Journal of minimally invasive gynecology 20 : $562-572$

9. Surapaneni K, Silberzweig JE (2008) Cesarean section scar diverticulum appearance on hysterosalpingography. AJR Am J Roentgenol 190: 870-874.

10. Vervoort AJ (2015) The HysNiche trial: hysteroscopic resection of uterine caesarean scar defect (niche) in patients with abnormal bleeding, a randomised controlled trial. BMC women's health 1: 103

11. Florio P, Filippeschi M, Moncini I, Marra E, Franchini M, et al. (2012) Hysteroscopic treatment of the cesarean-induced isthmocele in restoring infertility. Curr Opin Obstet Gynecol 24: 180-186.

12. Api M, Boza A, Gorgen H, Api O (2015) Should Cesarean Scar Defect Be Treated Laparoscopically? A Case Report and Review of the Literature. J Minim Invasive Gynecol 22: 1145-1152. 